2. A. Zygmund, Trigonometric series, 2nd ed., Vol. I, Cambridge Univ. Press, Cambridge, 1959.

3. G. Szegö, Orthogonal polynomials, Amer. Math. Soc. Colloq. Publ. Vol. 23, Amer. Math. Soc., Providence, R. I., 1939.

4. A. Hobson, The theory of spherical and ellipsoidal harmonics, Cambridge Univ. Press, Cambridge, 1931.

5. E. Madelung, Die mathematischen Hilfsmittel des Physikers, 6th ed., Springer, Berlin, 1957.

6. A. Rényi, Wahrscheinlichkeitsrechnung, VEB Deutscher Verlag der Wissenschaften, Berlin, 1962.

7. M. Loève, Probability theory, Van Nostrand, New York, 1955.

MATHEMATISCHES INSTITUT DER UNIVERSITÄT WIEN AND Statistical Laboratory, The Catholic University

\title{
A NOTE ON THE HAUSDORFF MOMENT PROBLEM
}

\author{
C. W. LEININGER
}

In [1, pp. 630-635], J. H. Wells presented a solution of the Hausdorff moment problem for the case of a quasicontinuous mass function. The purpose of this note is to extend that result to include Riemann-integrable mass functions.

If $\left\{d_{n}\right\}$ is a number sequence, let $A_{n p}=\left(\begin{array}{l}n \\ p\end{array}\right) \Delta^{n-p} d_{p}, n \geqq p, p=0,1$, $2, \cdots$ We observe that $[1$, p. 634 , Theorem $2.4($ ii) (b) $]$ may be stated as follows:

If $\epsilon>0$, there is a finite collection $C$ of nonoverlapping subsegments $(u, v)$ of the segment $(0,1)$ such that $\sum_{c}(v-u)=1$ and if $u<y<z$ $<v$, then there is a positive integer $N$ such that if $n>N$, $\left|\sum_{n y<p \leq n z} A_{n p}+\sum_{n y \leq p<n z} A_{n p}\right|<\epsilon$.

The arguments used to establish $[1$, p. 634, Theorem 2.4] and the associated theorems and lemmas [1, pp. 630-633] are readily modified to supply a proof of the following theorem.

THEOREM. If $\left\{d_{n}\right\}$ is a number sequence, the following two statements are equivalent:

(i) There is a function $g$ Riemann-integrable on $[0,1]$ such that $d_{n}=\int_{[0,1]} I^{n} d g, n=0,1,2, \cdots$;

Received by the editors October 15, 1964. 
(ii) (a) there is a number $M$ such that $\left|\sum_{p=0}^{k} A_{n p}\right|<M, 0 \leqq k \leqq n$, $n=0,1,2, \cdots$, and

(b) if $\epsilon>0$ and $0<\delta<1$, there is a finite collection $C$ of nonoverlapping subsegments $(u, v)$ of the segment $(0,1)$ such that $\sum_{c}(v-u)$ $>1-\delta$ and if $u<y<z<v$, then there is a positive integer $N$ such that if $n>N$,

$$
\left|\sum_{n y<p \leq n z} A_{n p}+\sum_{n y \leq p<n z} A_{n p}\right|<\epsilon .
$$

The crux of the matter lies in the observation that $[1$, p. 633 , Lemma 2.3] holds if the mass function is Riemann-integrable on $[0,1]$, and ${ }^{1}$ in noticing the following Ascoli-type result (compare with $[1$, p. 630, Theorem 2.1]):

LEMMA. Suppose $\left\{f_{n}\right\}$ is a uniformly bounded infinite sequence of real functions from $[0,1]$ and if $\epsilon>0$ and $0<\delta<1$, there is a finite collection $C$ of nonoverlapping subsegments $(u, v)$ of the segment $(0,1)$ such that $\sum_{c}(v-u)>1-\delta$ and if $u<y<z<v$, then there is a positive integer $N$ such that if $n>N$,

$$
\left|f_{n}(y)-f_{n}(z)\right|<\epsilon,
$$

and $\left\{g_{n}\right\}$ is an infinite subsequence of $\left\{f_{n}\right\}$ which converges at each point of a countable set which is dense in $[0,1]$. If, for each $x$ in $[0,1], h(x)$ is a cluster point of $\left\{g_{n}(x)\right\}$, then on $[0,1] h$ is Riemann-integrable and $\left\{g_{n}\right\}$ converges almost everywhere to $h$.

\section{REFERENCE}

1. J. H. Wells, Concerning the Hausdorff inclusion problem, Duke Math. J. 26 (1959), 629-645.

\section{Arlington State College}

1 The author is indebted to the referee for suggesting that the lemma be stated in the paper. 